\title{
Influence of Intravenous Sildenafil on Cerebral Oxygenation Measured by Near-Infrared Spectroscopy in Infants After Cardiac Surgery
}

\author{
NICOLE NAGDYMAN, THILO FLECK, BIRGIT BITTERLING, PETER EWERT, HASHIM ABDUL-KHALIQ, BRIGITTE STILLER, \\ MICHAEL HÜBLER, PETER E. LANGE, FELIX BERGER, AND INGRAM SCHULZE-NEICK \\ Departments of Paediatric Cardiology and Intensive Care [N.N., T.F., B.B., P.E., H.A.-K., B.S., P.E.L., I.S.-N.] and Cardiothoracic and \\ Vascular Surgery [M.H.], Deutsches Herzzentrum Berlin, 13353 Berlin, Germany; Department of Paediatric Cardiology [F.B.], \\ Charité-Universitätsmedizin Berlin, 13353 Berlin, Germany
}

\begin{abstract}
Sildenafil (Viagra) has been shown to be an effective pulmonary vasodilator and is increasingly used in patients with pulmonary hypertension. Its effects on the cerebral circulation are unclear and have not yet been described. We investigated the effect of i.v. sildenafil treatment on cerebral oxygenation in 13 children with elevated pulmonary vascular resistance due to congenital heart defects after cardiac surgery using near-infrared spectroscopy (NIRS). Median age was $4.5 \mathrm{mo}$, and median weight was $5.5 \pm 1.8$ $\mathrm{kg}$. Sildenafil was administered in three steps of $15 \mathrm{~min}$ each with cumulative doses of $0.025,0.1$, and $0.25 \mathrm{mg} / \mathrm{kg}$. We examined the changes of oxygenated hemoglobin $\left(\mathrm{HbO}_{2}\right)$, deoxygenated hemoglobin $(\mathrm{HHb})$, total hemoglobin $(\mathrm{tHb})$ concentration, cytochrome oxidase (CytOx) oxygenation, and cerebral tissue oxygenation index (TOI) in 13 children. A significant increase in cerebral $\mathrm{HbO}_{2}$ and $\mathrm{tHb}$ at the beginning of i.v. sildenafil administration with a decrease in $\mathrm{HHb}$ was observed. These changes led to a significant elevation in cerebral TOI from $63.4 \pm 2.5 \%$ to $65.7 \pm 2.8 \%$, whereas mean systemic arterial pressure and arterial oxygen partial pressure tended to decrease. In conclusion, we observed a reversible increase of $\mathrm{HbO}_{2}, \mathrm{tHb}$, and hemoglobin oxygen saturation in the scanned tissue section after i.v. sildenafil administration. These findings may be clinically relevant because they indicate that after cardiac surgery, sildenafil may increase cerebral blood flow $(\mathrm{CBF})$, probably due to general endothelial dysfunction after cardiopulmonary bypass (CPB). (Pediatr Res 59: 462-465, 2006)
\end{abstract}

$\mathrm{S}$ ldenafil is widely used in the treatment of erectile dysfunction and increasingly investigated as a therapy of pulmonary hypertension (1). Encouraging results have been shown in children with congenital heart defects and increased pulmonary vascular resistance after cardiac surgery $(2,3)$. Sildenafil is a highly selective inhibitor of the cyclic guanosine monophosphate (cGMP) degrading intracellular enzyme phosphodiesterase 5 (PDE 5). Nitric oxide (NO) activates a guanylate cyclase, which transforms guanylate triphosphate into cGMP, leading to smooth muscle relaxation and

Received June 15, 2005; accepted September 30, 2005.

Correspondence: Nicole Nagdyman, M.D., Department of Paediatric Cardiology and Intensive Care, Deutsches Herzzentrum Berlin, Augustenburger Platz1, 13353 Berlin, Germany; e-mail: nagdyman@dhzb.de

DOI: 10.1203/01.pdr.0000198772.26417.66 vasodilatation (4). PDE 5 is present in penile tissue, platelets, skeletal muscle, and vascular and visceral smooth muscle (5). The inhibition of PDE 5 results in an accumulation of cGMP, thus enhancing and prolonging the NO effect. Thus, PDE 5 inhibition in the lung leads to a reduction of pulmonary vascular resistance, decreased right ventricular afterload, and improved overall circulation. However, the NO-PDE 5 pathway is also involved in a multitude of cerebral functions and plays a central role in brain metabolism (6). PDE 5-mRNA has been detected in the mouse brain (5) and in human cerebral arteries (7); correspondingly, investigations in a rat model showed that sildenafil increases the brain levels of cGMP, evokes neurogenesis, and reduces neurologic deficits when given after stroke (8). In nonischemic rats, a transient increase in CBF was observed (8).

In humans, studies investigating the effect of sildenafil on $\mathrm{CBF}$ and oxygenation are rare $(7,9,10)$; although headache and confusion clearly are side effects, a case of intracerebral hemorrhage was reported after ingestion of sildenafil to improve sexual function (9). Studies in healthy subjects found no effect on CBF after oral sildenafil $(7,10)$. In view of the increasingly important use of sildenafil to treat various forms of pulmonary hypertension, the issue of its influence on cerebral perfusion, especially after $\mathrm{CPB}$, remains unresolved and of clinical importance, particularly in neonates and infants.

NIRS provides a continuous, noninvasive method to measure regional changes in tissue oxygenation (11) and is increasingly used to monitor intensive care patients (12-15). New generations of NIRS devices, such as the NIRO 300 (Hamamatsu Phototonics, Japan), use spatially resolved spectroscopy (SRS) to provide not only the usual measurements of changes in hemoglobin concentration, but to allow calculation of an absolute term, TOI, independent of a tissue path length factor $(16,17)$.
Abbreviations: CBF, cerebral blood flow; cGMP, cyclic guanyl monophosphate; CPB, cardiopulmonary bypass; CytOx, cytochrome oxidase; $\mathbf{H b O}_{2}$, oxygenated hemoglobin; HHb, deoxygenated hemoglobin; NIRS, near-infrared spectroscopy; NO, nitric oxide; PDE 5, phosphodiesterase 5; SRS, spatially resolved spectroscopy; $\mathbf{t H b}$, total hemoglobin; TOI, tissue oxygenation index 
Our objectives were the examination of cerebral oxygenation by NIRS in children after cardiac surgery while i.v. sildenafil was administered for elevated pulmonary vascular resistance.

\section{METHODS}

The study was approved by our Hospital Research Ethics Committee, and written informed consent was given by the parents.

Patients. We prospectively studied 13 patients (nine female, four male) with elevated pulmonary vascular resistance due to congenital heart defects after CPB. Median age was 4.5 mo (range, 2.1-20.9), and median weight was $5.5 \pm 1.8 \mathrm{~kg}$ (range, $3.5-10.6$ ). The underlying diagnoses were ventricular septal defects $(n=8)$, complete atrioventricular septal defects $(n=4)$, and complete abnormal pulmonary venous return $(n=1)$.

Study design. The subsequently described i.v. sildenafil administration was used in a study protocol to observe its short-term effects on pulmonary vascular resistance, for which ethical approval and written consent from the parents in all cases had been obtained. All children were intubated and ventilated mechanically. Sedation was maintained with continuous i.v. fentanyl $\left(4-20 \mu \mathrm{g} \cdot \mathrm{kg}^{-1} \cdot \mathrm{h}^{-1}\right)$ and midazolam $\left(4-20 \mu \mathrm{g} \cdot \mathrm{kg}^{-1} \cdot \mathrm{min}^{-1}\right)$. Continuous monitoring of heart rate, systemic arterial blood pressure, central venous pressure, and pulmonary artery pressure was performed invasively after cardiac surgery. The arteriovenous oxygen content difference $[\mathrm{avDO}$ $(\mathrm{mL} / \mathrm{L})]$ was calculated, and cardiac output was derived using the Fick principle as systemic oxygen consumption over $\mathrm{avDO}_{2}$. The study protocol started about 30 to $90 \mathrm{~min}$ after admission to the intensive care unit. When children were in a stable cardiorespiratory condition; baseline values for NIRS parameters were recorded for $10 \mathrm{~min}$.

Intravenous administration of sildenafil via a central venous catheter was performed at three different dose levels: S I, $0.025 \mathrm{mg} / \mathrm{kg}$; S II, $0.1 \mathrm{mg} / \mathrm{kg}$; S III, $0.25 \mathrm{mg} / \mathrm{kg}$. To provide near-steady state levels of each sildenafil dose, $80 \%$ of each dose was given as a bolus within $2 \mathrm{~min}$, and the remaining $20 \%$ were given for $15 \mathrm{~min}$ as maintenance dose. No change in medication other than that of sildenafil was performed during the study period. Arterial blood for blood gas analysis was taken at the end of each step of the study protocol. NIRS values and hemodynamic parameters were recorded continuously during the sildenafil administration. As measurement points for each step of the study protocol, we chose 1, 5, and 10 min after sildenafil administration. The median was calculated for the NIRS values at each step.

NIRS. This method using the NIRO 300 has been described in detail (15). Briefly, the NIRS probe was placed on the skin of the right forehead in the supraorbital region receiving reflected light from the frontal lobe. The emitter and receiver were fixed in a probe holder to ensure an interoptode distance of $40 \mathrm{~mm}$. The NIRS probe was combined with the measuring unit, which was connected to the NIRO 300 display unit. The NIRO 300 uses laser-emitting diodes to generate light at four different wavelengths $(775,810,847$, and 919 $\mathrm{nm})$. It employs the technique of SRS and uses multiple closely spaced detectors to measure light attenuation as a function of source-detector separation. From these measurements, it is possible to uncouple the absorption and scattering coefficients and combine these with an estimate of the wavelength dependence of light scattering (18). This SRS method, which was described in detail by Suzuki et al. (16) and Matcher et al. (17), allows the calculation of an absolute value for the TOI. In detail, the TOI describes the ratio of $\mathrm{HbO}_{2}$ to $\mathrm{tHb}$, which consists of $\mathrm{HbO}_{2}$ and $\mathrm{HHb}$ according to the formula calculated as $\mathrm{TOI}=\mathrm{HbO}_{2} /\left(\mathrm{HbO}_{2}+\mathrm{HHb}\right) \times 100$. The changes of chromophores $\left(\mathrm{HbO}_{2}, \mathrm{HHb}, \mathrm{tHb}, \mathrm{CytOx}\right)$ were calculated from the light attenuation changes at different wavelengths by using the modified Lambert-Beer law (11). NIRS values were recorded and saved to a floppy disk with a sample rate of $1 \mathrm{~s}$.

Statistics. All data were analyzed using SPSS 10.0 for Windows. The Wilcoxon signed rank test was used to compare the deviation from sildenafil administration to baseline values for TOI and the hemodynamic data. The changes of chromophore concentration of each measuring point were compared with zero set baseline values using a one-sample $t$ test. The data were presented as mean \pm standard error (SE). $p$ Values $<0.05$ were considered significant.

\section{RESULTS}

Effect of sildenafil on cardiorespiratory parameters. Mean arterial pressure, arterial oxygen partial pressure, and arterial oxygen saturation decreased significantly from baseline after the second and third step of sildenafil administration (Table 1). Heart rate, central venous oxygen saturation, and arterial carbon dioxide partial pressure remained stable throughout the protocol (Table 1). The cardiac index did not change significantly during the study protocol. There was no significant correlation between the cardiac index and cerebral TOI.

Effect of sildenafil on NIRS parameters. Changes in chromophores are presented in Figure 1. There was a fast increase in $\mathrm{HbO}_{2}$ and $\mathrm{tHb}$, whereas $\mathrm{HHb}$ decreased at the beginning of the first step of sildenafil infusion. These tendencies tended to wean off toward the end of the first step of the study protocol. A further significant increase in $\mathrm{HbO}_{2}$ and $\mathrm{tHb}$ with a decrease in $\mathrm{HHb}$ occurred again within the first minute of the second step of the study protocol. The deviation from baseline showed its maximal values for $\mathrm{HbO}_{2}(+\Delta 2.3 \pm 0.6 \mu \mathrm{mol} / \mathrm{L})$, $\mathrm{tHb}(+\Delta 0.9 \pm 0.2 \mu \mathrm{mol} / \mathrm{L})$ and $\mathrm{HHb}(-\Delta 1.3 \pm 0.4 \mu \mathrm{mol} / \mathrm{L})$. The changes of the chromophores toward the end of the second step and during the whole third step of sildenafil showed a tendency to return to baseline values, whereas tHb fell even below baseline values.

Intracellular CytOx redox state did not show any significant changes from baseline during the whole study. The TOI increased significantly with the beginning of sildenafil infusion in the first and second steps of the study protocol (Fig. 2). The increase at the second step of sildenafil administration where the TOI reached its maximal values $(65.7 \pm 2.8 \%)$ was significant compared with baseline as well compared with the end of the first step values $(p=0.01)$. The increase in tissue oxygenation, however, was transient, and the TOI values at the end of each step and at the whole third step of application did not differ significantly from baseline values.

\section{DISCUSSION}

The effect of sildenafil on cerebral oxygenation in children after cardiac surgery was studied using NIRS, a continuous, noninvasive method to measure regional changes in tissue oxygenation $(11,19)$. We observed a significant increase in cerebral $\mathrm{HbO}_{2}$ and tHb at the beginning of i.v. sildenafil administration and a concomitant decrease in $\mathrm{HHb}$. These changes suggested that, in children after CPB surgery, sildenafil may increase CBF.

The observed effects were in contrast to previous studies that found no effects of sildenafil on CBF and blood velocity in healthy subjects after oral administration $(7,10)$. In those studies, blood flow velocity was recorded in the middle cerebral artery by transcranial Doppler, and regional CBF in the perfusion area of the middle cerebral artery was measured using single photon emission computed tomography and ${ }^{133}$ xenon inhalation (7). We studied cerebral tissue oxygenation with NIRS, which gives information about the perfusion and oxygenation status of the smallest vessels and the capillary bed. Despite the different methods, we would expect that regional microvascular perfusion increases only in parallel with blood flow in the major inflow and outflow vessels.

However, sildenafil might directly affect neuronal cell metabolism, thus altering oxygen extraction and changes in oxyhemoglobin and hemoglobin oxygen saturation. This notion would be supported by the fact that PDE 5 is absent in 
Table 1. Cardiorespiratory data and TOI values of the patients at the different steps of sildenafil administration

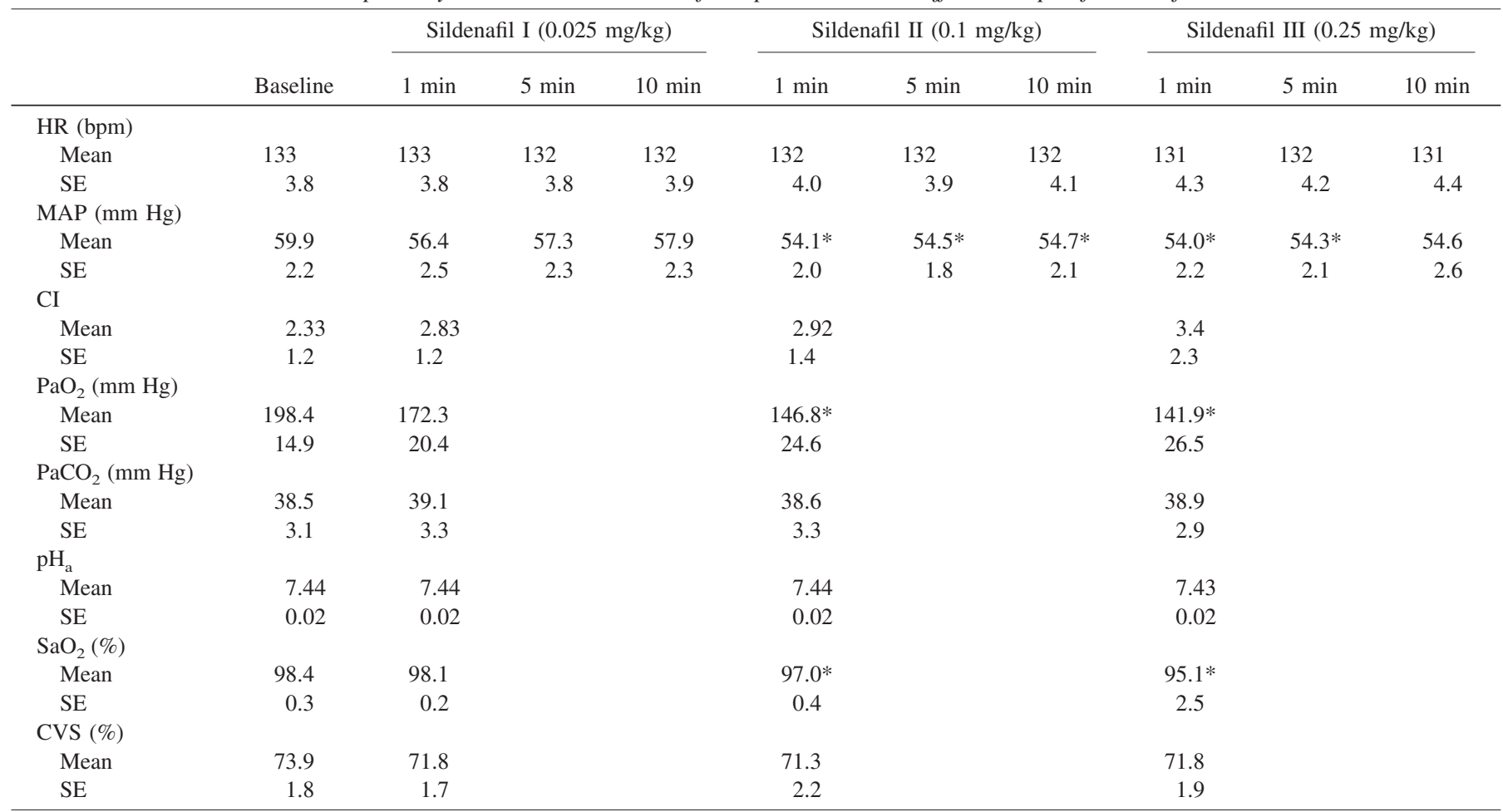

* $p$ Values compared with baseline values and different steps of sildenafil administration. $p$ Values $<0.05$ were considered to be significant.

$\mathrm{HR}$, heart rate; MAP, mean arterial pressure; CI, cardiac index; $\mathrm{PaO}_{2}$, arterial oxygen partial pressure; $\mathrm{PaCO}_{2}$, arterial carbon dioxide partial pressure; $\mathrm{pH}$, arterial $\mathrm{pH} ; \mathrm{SaO}_{2}$, arterial oxygenation saturation; $\mathrm{CVS}$, central venous oxygen saturation.

normal canine cerebral arteries (20) but present in various neuronal cell types of other species.

While it is known that cerebral vasodilation may also be a secondary phenomenon in response to decreasing arterial oxygen pressure, in our study, the significant decrease of the arterial $\mathrm{pO}_{2}$ observed after sildenafil administration did, however, not result in clinical hypoxemia (lowest arterial $\mathrm{pO}_{2} 141$ $\mathrm{mm} \mathrm{Hg}$ ). The observed decrease in arterial hemoglobin saturation and arterial oxygen partial pressure during our study

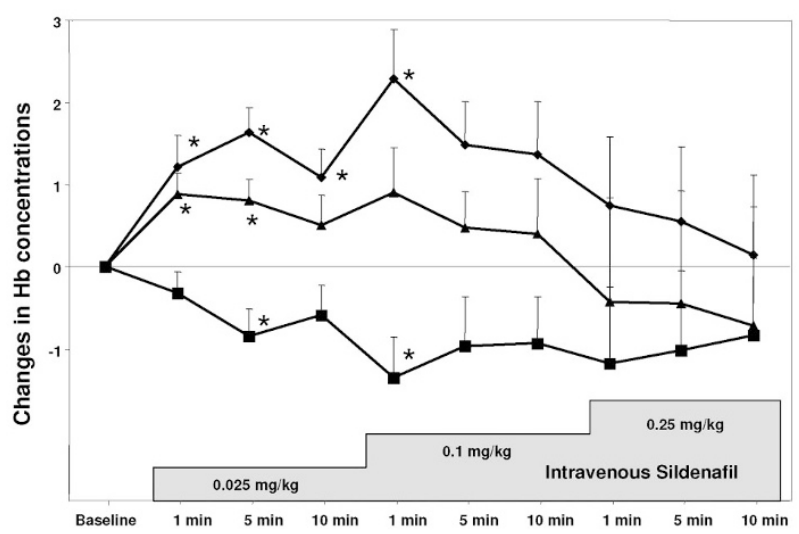

Figure 1. Chromophore concentration changes during the three steps of i.v. sildenafil application in children $(n=13)$ after cardiac surgery. At the beginning of the first and the second steps of sildenafil significant increase in $\mathrm{HbO}_{2}(\bullet)$ and $\mathrm{tHb}(\boldsymbol{\Lambda})$ and significant but transient decrease in $\mathrm{HHb}(\mathbf{\square})$ was observed. During the third step of application, values turned to baseline and showed no further significant changes. $p$ Value for comparison between baseline and different sildenafil steps. ${ }^{*} p$ Values $<0.05$ were significant. protocol was probably due to intrapulmonary shunting caused by a direct effect of sildenafil on the pulmonary arteries (21).

Also, the decrease in blood pressure, which is a well-known side effect of the vasodilator sildenafil (22), while being statistically significant, was clinically irrelevant in our patients and could not explain the changes in NIRS parameters. Finally, we could also exclude $\mathrm{PaCO}_{2}$ changes to be responsible for changes in $\mathrm{CBF}$ because we had constant $\mathrm{PaCO}_{2}$ values during the whole study.

Most importantly, the reason for the observed changes, however, was, in our opinion, because our protocol included

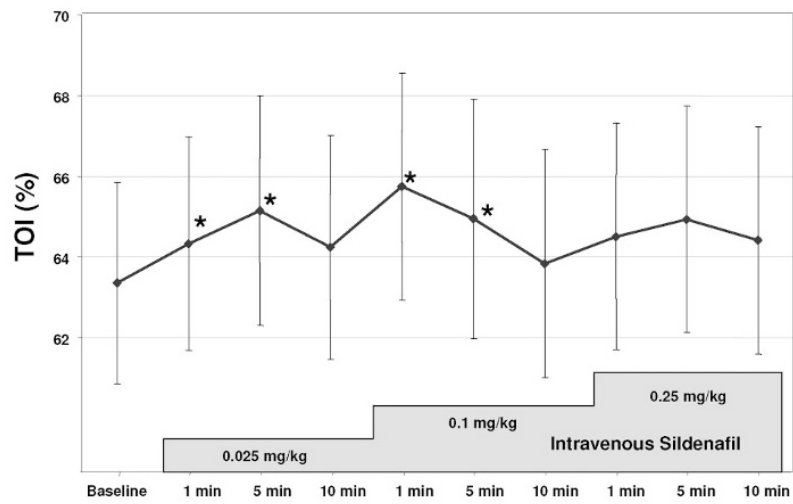

Figure 2. Changes of the TOI in 13 children during the different steps of sildenafil application. The TOI showed a significant increase from baseline at the beginning of the first two steps of sildenafil administration with a tendency to decrease toward the end of the steps. Changes at the third step were not significant. $p$ Value for comparison between baseline and different sildenafil steps. ${ }^{*} p$ Values $<0.05$ were significant. 
infants after CPB surgery, whereas the other studies examined healthy adults $(7,10)$. In children undergoing cardiac surgery, CPB causes general endothelial dysfunction, which may lead to endogenous NO depletion (23). Extending this finding to the situation with brain tissue as the best explanation at hand, enhancement in NO quantity might have an acute effect on the state of the cerebral vascular bed leading to vasodilation.

The reason why the increase in the TOI was only significant at the first and second steps of the study protocol and not at the final third step might be explained by a saturation effect on the PDE 5 obtained after the first two steps. In other vasoactive tissue such as the lung, we showed that low levels of sildenafil $(<250 \mathrm{ng} / \mathrm{mL})$ achieved a similar reduction in pulmonary vascular resistance comparable to high levels ( $>2000 \mathrm{ng} / \mathrm{mL})$, suggesting that lower sildenafil levels may cause an almost complete inhibition of the PDE 5 (21) and supporting the notion of a saturation effect of sildenafil at higher doses.

A limitation of NIRS monitoring was the fact that the changes in $\mathrm{HbO}_{2}, \mathrm{HHb}$, $\mathrm{tHb}$, and CytOx measured by Lambert-Beer law can be only measured as a relative deviation from the so-called baseline. However, the relative effects that we observed using NIRS were reproducible and interindividually and intraindividually measured at each step of the protocol and showed an acute response to the dose changes of sildenafil administration, thus implying a true response and not merely a shift or deviation from baseline. We studied only a small number of infants; however, we aimed to observe the effects of sildenafil on the cerebral vasculature in a proof-of principle study.

In conclusion, by observing cerebral oxygenation with noninvasive, continuous NIRS in children immediately after cardiac surgery, we observed a reversible increase in cerebral $\mathrm{HbO}_{2}$ and $\mathrm{tHb}$ after i.v. sildenafil, probably indicating an increased $\mathrm{CBF}$.

\section{REFERENCES}

1. Michelakis E, Tymchak W, Lien D, Webster L, Hashimoto K, Archer S 2002 Oral sildenafil is an effective and specific pulmonary vasodilator in patients with pulmonary arterial hypertension: comparison with inhaled nitric oxide. Circulation $105: 2398-2403$

2. Abrams D, Schulze-Neick I, Magee AG 2000 Sildenafil as a selective pulmonary vasodilator in childhood primary pulmonary hypertension. Heart 84:E4-
3. Atz AM, Lefler AK, Fairbrother DL, Uber WE, Bradley SM 2002 Sildenafil augments the effect of inhaled nitric oxide for postoperative pulmonary hypertensive crises. J Thorac Cardiovasc Surg 124:628-629

4. Corbin JD, Francis SH 1999 Cyclic GMP phosphodiesterase-5: target of sildenafil. J Biol Chem 274:13729-13732

5. Wallis RM, Corbin JD, Francis SH, Ellis P 1999 Tissue distribution of phosphodiesterase families and the effects of sildenafil on tissue cyclic nucleotides, platelet function, and the contractile responses of trabeculae carneae and aortic rings in vitro. Am J Cardiol 83:3C-12C

6. Zanzinger J 2002 Mechanism of action of nitric oxide in the brain stem: role of oxidative stress. Auton Neurosci 28:24-27

7. Kruuse C, Thomsen LL, Jacobsen TB, Olesen J 2002 The phosphodiesterase 5 inhibitor sildenafil has no effect on cerebral blood flow or blood velocity, but nevertheless induces headache in healthy subjects. J Cereb Blood Flow Metab 22:1124-1131

8. Zhang R, Wang Y, Zhang L, Zhang Z, Tsang W, Lu M, Chopp M 2002 Sildenafil (Viagra) induces neurogenesis and promotes functional recovery after stroke in rats. Stroke 33:2675-2680

9. Monastero R, Pipia C, Camarda LK, Camarda R 2001 Intracerebral hemorrhage associated with sildenafil citrate. J Neurol 248:141-142

10. Arnavaz A, Aurich A, Weissenborn K, Hartmann U, Emrich HM, Schneider U 2003 Effect of sildenafil (Viagra) on cerebral blood flow velocity: a pilot study. Psychiatry Res 122:207-209

11. Wahr JA, Tremper KK, Samra S, Delpy DT 1996 Near-infrared spectroscopy: theory and applications. J Cardiothorac Vasc Anesth 10:406-418

12. Adcock LM, Wafelman LS, Hegemier S, Moise AA, Speer ME, Contant CF, Goddard-Finegold J 1999 Neonatal intensive care applications of near-infrared spectroscopy. Clin Perinatol 26:893-903

13. Schulz G, Weiss M, Bauersfeld U, Teller J, Haensse D, Bucher HU, Baenziger O 2002 Liver tissue oxygenation as measured by near-infrared spectroscopy in the critically ill child in correlation with central venous oxygen saturation. Intensive Care Med 28:184-189

14. Abdul-Khaliq H, Troitzsch D, Schubert S, Wehsack A, Bottcher W, Gutsch E, Hubler M, Hetzer R, Lange PE 2002 Cerebral oxygen monitoring during neonatal cardiopulmonary bypass and deep hypothermic circulatory arrest. Thorac Cardiovasc Surg 50:77-81

15. Nagdyman N, Fleck T, Barth S, Abdul-Khaliq H, Stiller B, Ewert P, Huebler M, Kuppe H, Lange PE 2004 Relation of cerebral tissue oxygenation index to central venous oxygen saturation in children. Intensive Care Med 30:468-471

16. Suzuki S, Takasaki S, Ozaki T, Kobayashi Y 1999 A tissue oxygenation monitor using NIR spatially resolved spectroscopy. Proc SPIE 3579:144-145

17. Matcher SJ, Kirkpatrick P, Nahid K, Cope M, Delpy DT 1995 Absolute quantification methods in tissue near infrared spectroscopy. Proc SPIE 2389:486-495

18. Thavasothy M, Broadhead M, Elwell C, Peters M, Smith M 2002 A comparison of cerebral oxygenation as measured by the NIRO 300 and the INVOS 5100 nearinfrared spectrophotometers. Anaesthesia 57:999-1006

19. Jobsis FF 1977 Noninvasive, infrared monitoring of cerebral and myocardial oxygen sufficiency and circulatory parameters. Science 198:1264-1267

20. Inoha S, Inamura T, Ikezaki K, Nakamizo A, Amano T, Fukui M 2002 Type V phosphodiesterase expression in cerebral arteries with vasospasm after subarachnoid hemorrhage in a canine model. Neurol Res 24:607-612

21. Schulze-Neick I, Hartenstein P, Li J, Stiller B, Nagdyman N, Hübler M, Butrous G, Petros A, Lange P Redington AN 2003 Intravenous sildenafil is a potent pulmonary vasodilator in children with congenital heart disease. Circulation 108:II167-II173

22. Jackson G, Benjamin N, Jackson N, Allen MJ 1999 Effects of sildenafil citrate on human hemodynamics. Am J Cardiol 83:13C-20C

23. McMullan DM, Bekker JM, Parry AJ, Johengen MJ, Kon A, Heidersbach RS, Black SM, Fineman JR 2000 Alterations in endogenous nitric oxide production after cardiopulmonary bypass in lambs with normal and increased pulmonary blood flow. Circulation 102:III172-III178 